\title{
Edgeless and slim-edge solutions for silicon pixel sensors
}

\author{
Gian-Franco Dalla Betta ${ }^{1}$, Marco Povoli \\ University of Trento and INFN \\ Via Sommarive, 9 - 38123 Povo di Trento, Italy \\ E-mail: gianfranco.dallabetta@unitn. it
}

\section{Maurizio Boscardin}

Fondazione Bruno Kessler (FBK),

Via Sommarive, 18, - 38123 Povo di Trento, Italy

\section{Gregor Kramberger}

Jožef Stefan Institute (JSI),

Jamova 39, SI-1000 Ljubljana, Slovenia

\begin{abstract}
Applications of silicon pixel sensors in future HEP experiments (e.g., at the HL LHC) are setting increasing demands on the minimization of the dead area at the edge, calling for adequate design/technological solutions for edge termination. In this respect, significant advantages are offered by the adoption of 3D fabrication technologies, which allow for a wider choice of edge designs (among them, active edges) owing to the use of the third dimension within the sensor substrate. This paper reviews the most interesting approaches to edgeless and slim-edge sensors, with emphasis on the original solutions developed by Fondazione Bruno Kessler (Trento, Italy) in collaboration with the University of Trento and INFN in the past few years. In particular, planar sensors with active edge and double-sided 3D sensors with slim-edge are discussed. The main design and technological issues will be reported, as well as selected results from numerical device simulations and electrical/functional tests performed before and after irradiation.
\end{abstract}

The 22nd International Workshop on Vertex Detectors

16-20 September 2013

Lake Starnberg, Germany

\footnotetext{
${ }^{1}$ Speaker
} 


\section{Introduction}

The edge termination is one of the most critical aspects in the design of silicon radiation sensors. Regardless of the sensor type (e.g., pixel, strip, pad, etc.), the active area is normally surrounded by a main guard-ring, to be kept at the same voltage as the read-out electrodes, in order to collect the edge leakage current and to ensure a uniform electric field distribution with respect to the innermost regions of the active area. Multiple floating guard rings are also often used around the main guard ring in order to obtain a higher breakdown voltage and a better long-term stability (see [1] and references therein). Additionally, between the guard ring(s) and the cut line, a safety distance is normally present, so that the depletion region spreading from the active area can not reach the physical edge of the sensor, thus minimizing the risk of leakage current injection from the defects introduced by detector dicing with diamond saws. The size of the features mentioned above of course depend on a number of parameters specific to the considered application: among them, the substrate thickness and doping concentration, the oxide charge density, the irradiation scenario and the required bias conditions. In all cases a relatively wide insensitive region is introduced at the sensor edge, the size of which can span from a few hundreds of micrometers to more than $1 \mathrm{~mm}$. As a result, within a tracking system, sensor overlap within the same layer has been normally used, that complicates the detector assembly and increases the material budget.

In the past few years, there has been an increasing interest to edgeless sensors for future experiments in particle colliders and other applications. As an example, the High-Luminosity LHC upgrade (HL-LHC), because of material budget restrictions and tight mechanical constraints, call for new sensors for the inner layers (pixels) with reduced geometrical inefficiency [2]. This trend has already started with the ATLAS Insertable B-Layer (IBL), which set a maximum dead region of $225 \mu \mathrm{m}$ along the direction parallel to the beam at the edge of single-chip sensors [3]. Moreover, the TOTEM experiment uses 1-side edgeless strip sensors [4], and other foreseen forward physics experiments, like the AFP [5], plan to use 1-side edgeless pixel sensors to maximize their physics potential. Edgeless sensors are also appealing for X-ray imaging applications: 4-side edgeless sensors allow for seamless large area images by tiling several detector modules into matrices [6], whereas 1-side edgeless sensors are sufficient to largely improve the detection efficiency for X-ray imaging in the edge-on configuration [7].

Among other edgeless approaches, of particular interest are those based on 3D fabrication technologies, which offer more design flexibility owing to the possibility to exploit the third dimension within the sensor substrate. In fact, it was in the framework of our 3D sensor projects that we have started to investigate edgeless solutions a few years ago, and we will here report on some recent results from both planar and 3D sensors.

This paper is organized as follows: in Section 2, the most interesting approaches so far proposed for edgeless or slim-edge sensors are recalled; in Section 3, recent results relevant to the R\&D activities carried out in this field by Fondazione Bruno Kessler (FBK, Trento, Italy) in collaboration with the University of Trento and INFN are reviewed, with emphasis on planar sensors with active edges and double-sided 3D sensors with slim-edge. Conclusions are drawn in Section 4. 


\section{Different approaches to edgeless sensors}

Several technological and design solutions have already been reported to minimize the insensitive area at the sensor edge. One of the first methods adopted has been laser dicing, in combination with chemical etching of the edge and/or aging treatments to reduce the cut damage [8]: while providing a significant improvement compared to standard saw dicing, results were not optimal in terms of leakage current and stability. Another interesting solution consists of a current terminating ring (CTR) used in combination to a normal bias ring [4]: the CTR is able to sink most of the high leakage current caused by saw dicing, while only a small fraction of this current $(\sim 0.1 \%)$ is drawn by the bias ring. By doing so, a slim edge as small as $60 \mu \mathrm{m}$ was obtained for the TOTEM experiment.

It was mentioned that the ATLAS IBL called for a slim edge of $225 \mu \mathrm{m}$. While for 3D sensors a dedicated 3D termination was designed, that will be described in Section 3.2, for planar sensors this goal was achieved by optimizing the guard ring design and by partially overlapping the outermost, $500 \mu \mathrm{m}$ long pixels to the guard ring structure [3]. It should be noticed that this is possible for "n-on-n" sensors, where pixels and guard rings lay on opposite sides of the wafers; " $n$-on-p" sensors, that are also considered as an option for HL-LHC because of their lower cost, offer a lower design flexibility for the edge region, because the guard rings are on the same side as the pixels, thus calling for alternative solutions.

\subsection{Active edge technologies}

A major breakthrough came in 2001 with the so-called "active edge", which was introduced at the Stanford Nanofabrication Facility (SNF) as an extension of 3D detector technology [9], but was later applied also to planar sensors [10]. Active edges consist of deep trenches that are etched by Deep Reactive Ion Etching (DRIE) around the sensor active area and are then doped to act as (normally ohmic) electrodes. By doing so, full signal sensitivity up to a few micrometers from the physical edge can be obtained, as demonstrated by tests with X-rays and particle beams [11], [12], [13].

Sensor dicing and related problems are avoided with active edges; moreover, arbitrary edge shapes can be achieved. This comes at the expense of an increased process complexity, due to several DRIE steps and other critical features. In fact, a support wafer is initially fusion bonded to the sensor wafer [14], in order to hold different dice together once the trenches have been etched [9], and should finally be removed. While wafer bonding is nowadays a reliable technology, support wafer removal is quite challenging in the presence of etched trenches. Furthermore, in order to ease detector assembly within a system, the substrate bias should preferably be applied from the sensor back side. To this purpose, after support wafer removal, the residual insulating layers on the back side have to be etched and a metal layer has to be deposited. Extensive R\&D is on going (e.g., by using temporary wafer carriers) to engineer these back-end steps and find the most appropriate combinations with the sensor interconnect processes.

Besides the original proponents at SNF, active edge technology has also been developed at other processing facilities, like those of the Norwegian Foundation for Scientific and Industrial Research (SINTEF) [15], FBK [16], and the Technical Research Centre of Finland 
(VTT) [17]. In particular, the latter has developed an original process on 6-inch wafers, including four-quadrant edge implantation and successfully mastering the above-mentioned back side issues [18]. Among recent results obtained from VTT edgeless pixel sensors, particularly impressive are those relevant to a very large area, Timepix-based X-ray detector system [6]. Moreover, edgeless pixel sensors from VTT, compatible with the ATLAS pixel read-out chips, have been used to develop four-side buttable modules using vertical integration technologies in view of the ATLAS Pixel system upgrade: very good results in terms of electrical characteristics and charge collection properties have been obtained, with edge pixels showing performance comparable to the central ones, both before irradiation and after irradiation up to $5 \times 10^{15} \mathrm{n}_{\mathrm{eq}} / \mathrm{cm}^{2}$ [19]. Within the ATLAS Planar Pixel Sensor Collaboration, also planar edgeless pixels from FBK are being studied with encouraging results [20]. Even after irradiation at large fluences, the breakdown voltage was found to be high enough to obtain good charge collection efficiency [21].

Other structures fabricated with dedicated technologies without the use of a support wafer are worth mentioning. Trenches partially etched and doped from one side, combined with different types of guard rings and a final dicing on the opposite side, were reported in [22] and proved to yield an effective slim-edge of about $50 \mu \mathrm{m}$. For X-ray sensors to be used in the edgeon configuration, a 1-side full trench was demonstrated in [7], with a good mechanical stability ensured by the three non-etched sides.

\subsection{Scribe-Cleave-Passivate technique}

The Scribe-Cleave-Passivate (SCP) technique represents an interesting alternative for slim edges [23]. Since all treatments can be applied post-processing and at low temperatures, it is compatible with any kind of sensor, regardless of the specific technology, with the only requirement to have wafers with $<100>$ crystal orientation with reasonably good alignment between sensor and lattice in order to obtain good quality rectangular side cleaving. Wafer scribing and cleaving are used to minimize the number of defects with respect to a standard dicing saw, whereas edge surface passivation aims at allowing for a bias voltage gradient along the sidewall surface. The SCP technique actually includes a variety of methods: the classic diamond-stylus scribing step can be replaced with laser dicing, or by etching (e.g, by $\mathrm{XeF}_{2}$ or DRIE); the cleave step can be enhanced by sidewall damage removal by a reactive agent; the passivation can be performed with different dielectric layers (e.g., native oxide, oxide or nitride from Plasma Enhanced Chemical Vapor Deposition), to be tailored to different substrate doping types in terms of interface charge sign. In particular, for p-type substrates, negative charge is required, and it can be obtained with alumina $\left(\mathrm{Al}_{2} \mathrm{O}_{3}\right)$ by Atomic Layer Deposition [23].

In the past few years, different SCP options have been explored with promising results, also in terms of charge collection uniformity in the edge region compared to the core of the sensors [24]. Recent work has been mainly focused on: i) wafer-level processing, in order for the manual methods so far adopted to be automatized for volume productions; ii) radiation hardness, that is a possible concern particularly for $\mathrm{Al}_{2} \mathrm{O}_{3}$ passivated devices, since high edgecurrents have been observed after irradiation at low fluences [24]. 
The slim-edge post-processing based on DRIE $+\mathrm{Al}_{2} \mathrm{O}_{3}$ passivation, recently proposed by Hamamatsu for p-type sensors, and somehow inspired to SCP, is also worth consideration [25].

\section{Recent results from $R \& D$ activities in Trento}

\subsection{Planar sensors with active edges}

The first batch of planar sensors with active edges (p-on-n) was fabricated at FBK in 2010, in order to assess the critical steps of the technology and to study the impact of the edge region design on the electrical and functional characteristics of the devices [16]. Devices were made on Float Zone (FZ) $200 \mu \mathrm{m}$ thick n-type substrates, fusion bonded at SINTEF to $300 \mu \mathrm{m}$ thick support wafers. The trenches were etched by DRIE with $\sim 10 \mu \mathrm{m}$ width, doped by phosphorus diffusion and then filled with poly-Si to cope with the severe topography on the etched side for subsequent lithographical steps. Details on the fabrication process can be found in [16].

On test diodes, we have thoroughly investigated the trade-off between the breakdown voltage and the edge sensitivity as a function of the distance (gap) between the outermost junction and the ohmic active edge, while exploring different field limiting options (e.g., field plates, floating rings). As expected, the breakdown voltage was found to increase with the gap, ranging from $\sim 100 \mathrm{~V}$ at $10 \mu \mathrm{m}$ to $\sim 300 \mathrm{~V}$ at $150 \mu \mathrm{m}$ [26]. Field plates are effective to increase the breakdown voltage (by more than $100 \mathrm{~V}$ ) for gaps larger than $20 \mu \mathrm{m}$, in good agreement with numerical device simulations. Selected diodes have been functionally characterized by means of laser and X-ray beam scans, and proved to work properly with a high efficiency up to a few micrometers from their physical edge [27].
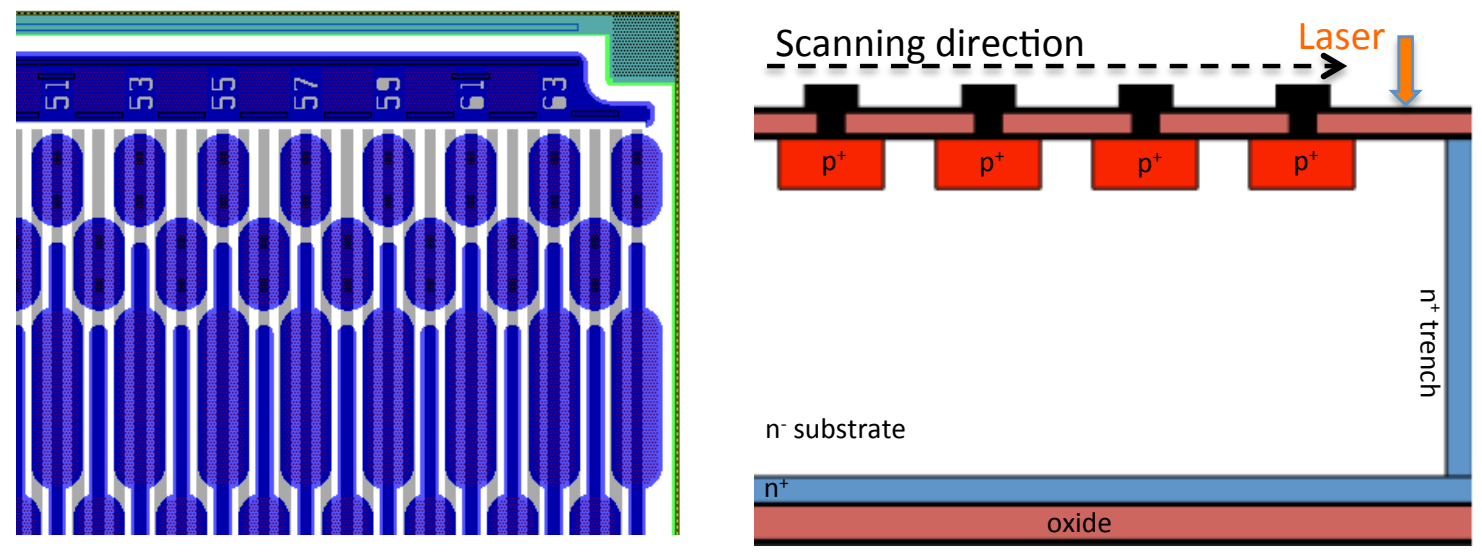

Figure 1 Layout detail (left) and schematic cross-section (right) of a planar strip sensor with active edge.

Recently, we have further tested the edge response in collaboration with the Jožef Stefan Institute (JSI, Ljubljana, Slovenia), also including radiation effects. As a test vehicle, we have used strip sensors, featuring a read-out strip pitch of $50 \mu \mathrm{m}$. Fig. 1 shows a detail of the layout and a schematic cross-section of the sensors. The nominal distance between the center of the outermost strip and the active edge (trench) is $50 \mu \mathrm{m}$. All strips were shorted and read-out like a diode with a fast amplifier. Two sensors were characterized with a scanning laser setup (Fig.1 also shows the scanning direction, with laser beam impinging perpendicularly to the surface): 
one sensor (S3) was not irradiated, whereas the other one (S13) was irradiated with reactor neutrons at JSI at a fluence of $2 \times 10^{15} \mathrm{n}_{\mathrm{eq}} / \mathrm{cm}^{2}$ and annealed for 80 minutes at $60^{\circ} \mathrm{C}$ before testing. Two laser wavelengths were used: i) $658 \mathrm{~nm}$ (red), with a penetration depth in $\mathrm{Si}$ of about $3 \mu \mathrm{m}$ and a beam Full Width at Half Maximum (FWHM) of $6 \mu \mathrm{m}$, and ii) $1064 \mathrm{~nm}$ (IR), with a penetration depth in Si larger than $1 \mathrm{~mm}$ (thus emulating a m.i.p. in a $200 \mu \mathrm{m}$ thick sensor) and a beam FWHM of $9 \mu \mathrm{m}$.

Fig. 2 shows the collected charge (calculated as the integral of the current pulses over 60 $\mathrm{ns}$ ) as a function of the position for the non-irradiated strip sensor S3 at different bias voltages. The nominal position of the trench is also indicated (note that signals can be observed when the trench is hit and also slightly outside it due to the finite width of the laser beam). The valleys in the data curves are due to the light reflection from the metal layers. It can be seen that the active edge region is efficient at very low voltage, i.e., lateral depletion (electric field) is established soon. For the red laser, increasing the voltage does not change charge collection near the edge, whereas the effect is much stronger for the IR laser, as expected, since depletion deep in the substrate is established at higher voltages. For the same reason, close to the edge larger voltage is required for the same collected charge. For the red (IR) laser, the active edge affects the response of the adjacent strips up to $\sim 300$ (200) $\mu \mathrm{m}$ away from it, after that the charge collection is as expected for a simple pad detector.
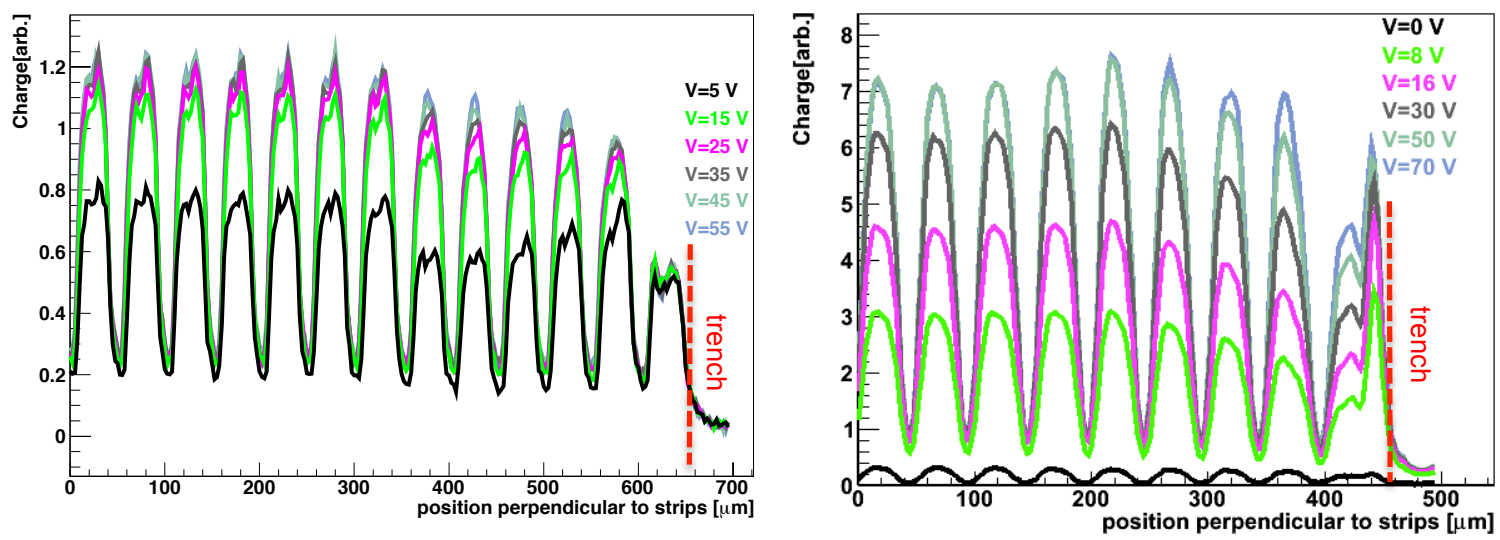

Figure 2 Collected charge as a function of the position for the non-irradiated strip sensor S3 tested with scanning laser setup at different bias voltages: red laser (left) and IR laser (right).

In comparison, Fig. 3 shows the data relevant to the irradiated sensor S13. Note that the considered fluence is high enough to cause substrate type-inversion, so low field at the p-type strips, and non-negligible charge trapping effects, hence the relatively small collected charge with respect to the pre-irradiation case. The active edge affects the response a few strips away $(\sim 200 \mu \mathrm{m})$ also after irradiation, but with an opposite trend (i.e., decreasing charge as the distance from the edge increases): this is due to the strong lateral electric field induced by the ntype trench in the type-inverted substrate, as also predicted by device simulations. This is particularly evident for the red laser, since the electric field is very strong close to the surface where $658 \mathrm{~nm}$ photons are absorbed. In particular, the large increase of charge from $150 \mathrm{~V}$ to $170 \mathrm{~V}$ at the edge, but not in the center of the detector, is probably due to multiplication, since the field is high enough to cause breakdown at the same voltage, as observed from the currentvoltage curves. For the IR laser, the increase of the collected charge with voltage is more pronounced, as the electric field grows in a larger part of the detector where charge is generated, 
whereas the increase of the charge at high voltage $(150-170 \mathrm{~V})$ is not so big at the edge as for red laser, since only a small fraction of the charge is generated in the very high electric field region for IR.
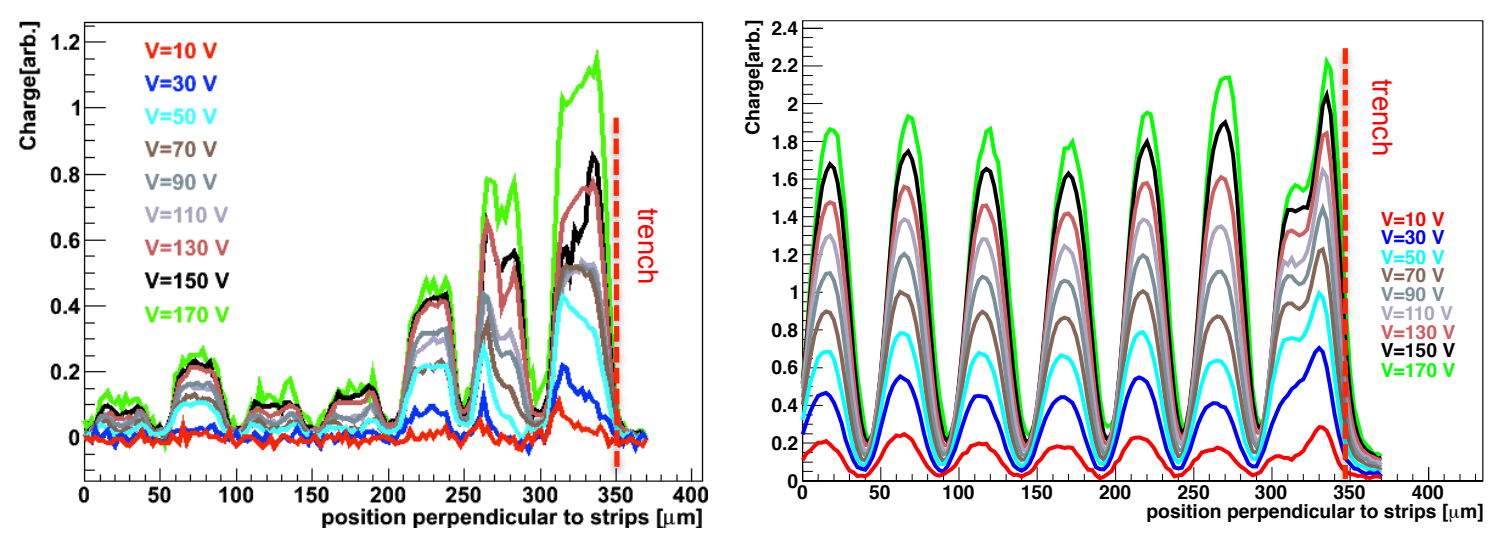

Figure 3 Collected charge as a function of the position for the irradiated strip sensor S13 tested with scanning laser setup at different bias voltages: red laser (left) and IR laser (right).

\subsection{Slim edges in double-sided 3D sensors}

Due to a simpler fabrication technology, as compared to the original one developed at SNF, double-sided 3D sensors from FBK and from the Centro Nacional de Microelectronica (CNM) in Barcelona, Spain, offer some advantages in terms of processing time and yield, so they were chosen for the production of the ATLAS IBL 3D pixel sensors [15]. However, active edges are not compatible with a double-sided process without a support wafer, so different solutions had to be found for the required slim edge. In CNM sensors the slim edge includes a 3D guard ring, aimed at sinking the leakage current originating from the cut line, and is surrounded by a double fence of ohmic columns [15]. In FBK sensors, no guard ring is present, and the slim edge consists of a multiple ohmic column termination, biased at the same substrate potential. As a result, the depletion region spreading from the outermost junction column is blocked within a short distance and does not reach the cut-line, so that no edge leakage current is drawn by the detector [28]. This solution proved to be effective for the IBL sensors, both before and after irradiation [3]: in fact, the $\sim 200 \mu \mathrm{m}$ ohmic fence was found to be oversized and the proposed slim-edge concept can be improved to reduce the dead area to $100 \mu \mathrm{m}$ or less, as demonstrated with some aggressive dicing tests oriented to the AFP project [29].

Deep insight into the slim-edge behavior was gained from functional measurements of $3 \mathrm{D}$ diodes using position resolved laser and X-ray beam tests. In particular, using a read-out made of a charge amplifier and a fast shaper (20 ns), so as to suppress any diffusion-related charge collection, it was possible to monitor the lateral spread of the depletion region. As an example, Fig. 4 shows the map of the signal measured with a $1060 \mathrm{~nm}$ laser beam (FWHM $\sim 11 \mu \mathrm{m}$ ) in a diode biased at $15 \mathrm{~V}$, i.e., beyond full depletion [15]. For a direct comparison, the slim-edge layout and the simulated hole-density distribution at the same voltage are also shown in Fig. 4. The $\mathrm{n}^{+}$columns are easily recognized in the signal map as the low signal regions on the right, due to the light reflection from the metal layers. The high signal region spreads from around the 
$\mathrm{n}^{+}$columns and, in the slim edge region, it extends slightly beyond the first row of ohmic columns, in good agreement with the simulated depletion region. Even at the largest bias voltage allowed by sensor breakdown $(\sim 50 \mathrm{~V})$, the depletion region width would be only slightly larger than that shown in Fig. 4, so that the slim-edge size could be reduced to about $100 \mu \mathrm{m}$.
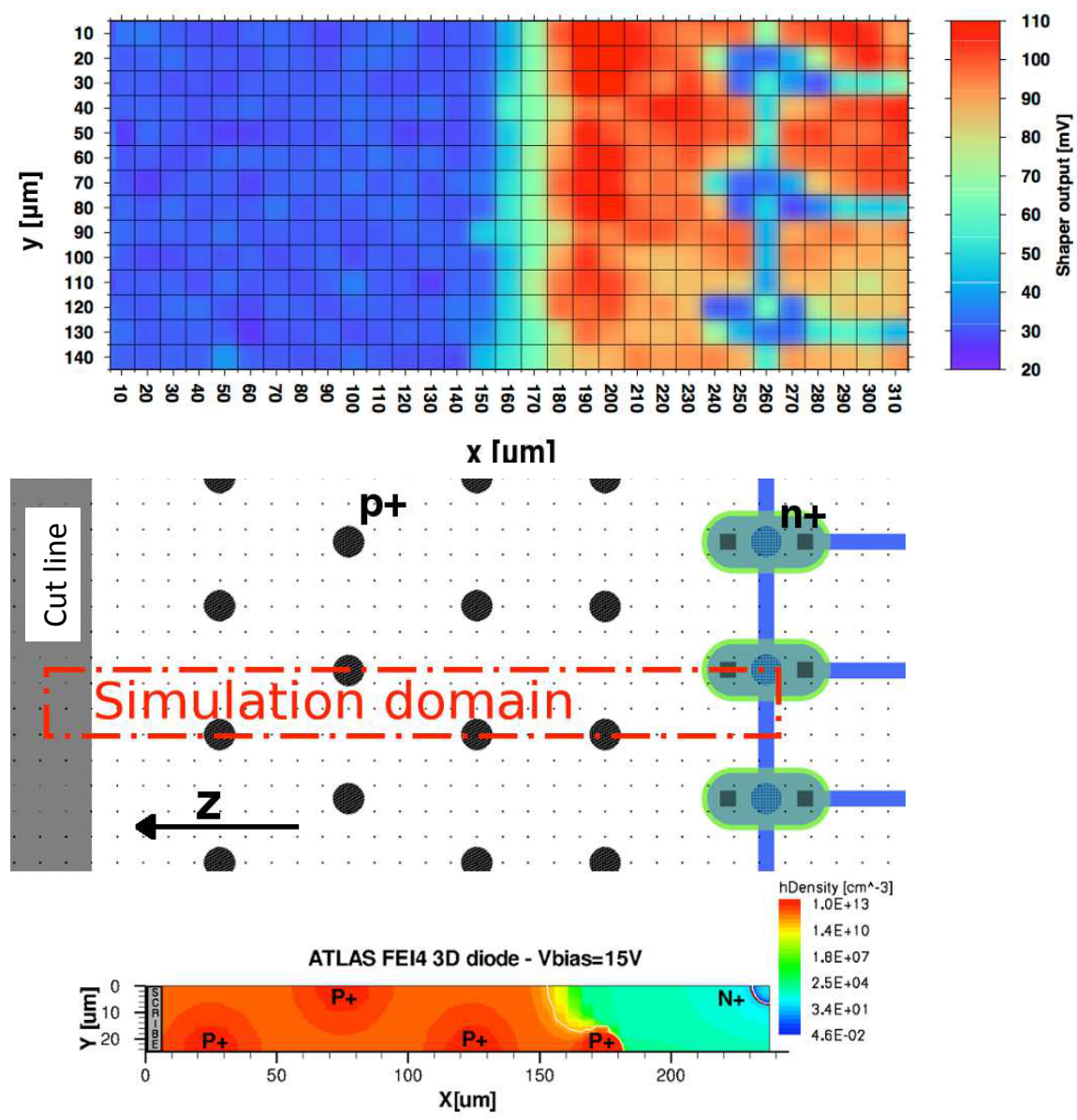

Figure 4 Results from position resolved laser scan on the slim-edge region of a $3 \mathrm{D}$ diode biased at $15 \mathrm{~V}$, compared to device simulations: top) $2 d$ signal map; center) diode layout and simulation domain; bottom) simulated hole density along an horizontal plane showing the lateral depletion region extension.

An even better confinement of the depletion region can be achieved by replacing columnar electrodes with dashed trenches within the ohmic fence (see layout in Fig. 5). This solution was studied with the aid of simulations [26] and, after the IBL production, implemented in an R\&D batch of 3D double-sided sensors at FBK [30]. It could be applied with minor process complications also to planar sensors. The $\mathrm{p}^{+}$dashed trenches are etched by the same DRIE step used to etch the $\mathrm{p}^{+}$columns and doped by the same boron diffusion step. Using the same test setup described above (position resolved IR laser with fast read out), it was possible to monitor the lateral spread of the depletion region also in this case. As an example, Fig. 5 shows the map of the signal measured in a diode biased at $60 \mathrm{~V}$, which features low and high signal regions. Low signals are observed from within the active area (due to light reflection from the metal) and 
from the non depleted region beyond the dashed trenches and close to the cut line. High signals are observed from the metal-free square windows within the active area and from the depleted region spreading from the $\mathrm{n}+$ column and stopping at the dashed trenches. Notably, the high signal profile within the slim-edge region well matches the layout of the dashed trenches. With this improved slim-edge design, the dashed trenches can be placed much closer to the cut line, so that the dead area at the edge can be decreased to about $50 \mu \mathrm{m}$, which is good enough for many applications [29].
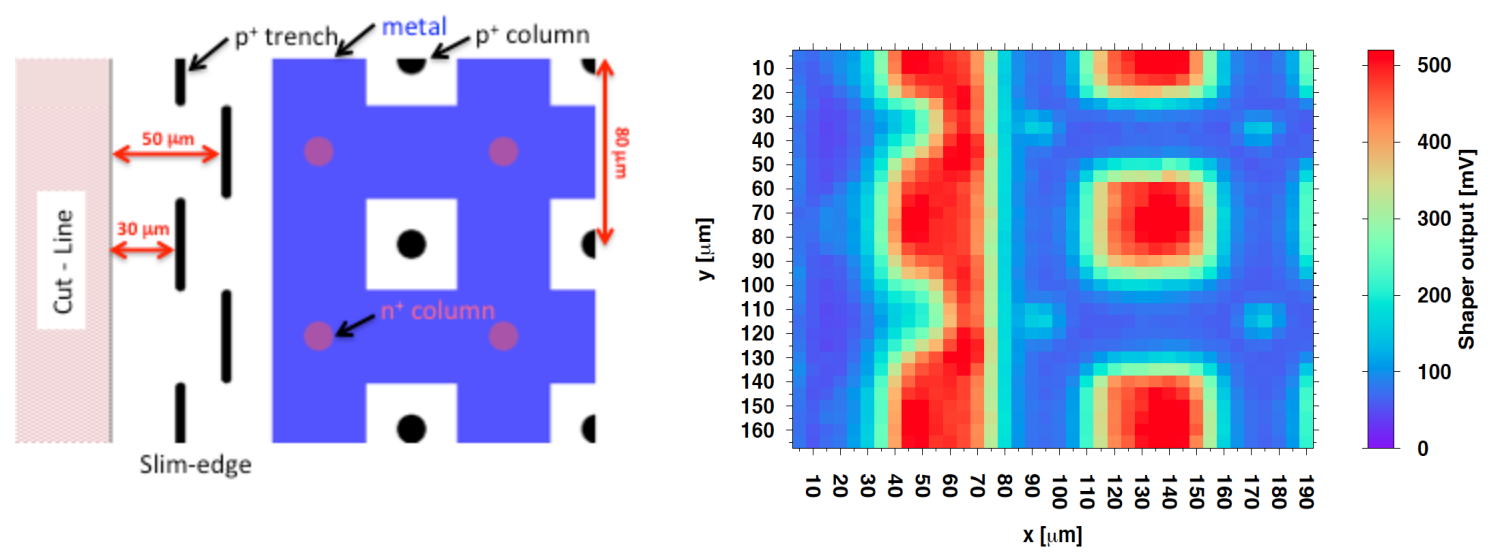

Figure 5 Layout detail (left) and position resolved laser scan (right) for a 3D diode with an improved slim-edge based on dashed trenches, measured at $60 \mathrm{~V}$ bias voltage.

\section{Conclusions}

In the past few years, pushed by the increasing needs of future experiments in high luminosity colliders and of X-ray imaging applications, important progress has been made in edgeless and slim-edge pixel sensors. In this paper, we have reviewed the most interesting approaches, and reported some recent results from the $\mathrm{R} \& \mathrm{D}$ activities going on in Trento.

Active edges were derived from 3D sensor technology. They provide the best results in terms of minimization of the dead area, at the expense of process complication. Directions for future work include more cost-effective technological solutions, reliable strategies for the integration in large detector systems, and thorough assessment of the radiation tolerance. The SCP technique is a very appealing alternative due to its flexibility, but the device behavior after irradiation requires further investigations, and large scale industrial feasibility is still a concern. For double-sided 3D sensors, a very slim edge $(\sim 50 \mu \mathrm{m})$, dashed-trench design has been demonstrated on small size 3D diodes, and will be applied to larger detectors for further testing.

\section{Acknowledgements}

This work has been partially funded by: (i) the Provincia Autonoma di Trento through the Project MEMS2; (iii) the Italian National Institute for Nuclear Physics (INFN) through the Projects TREDI and TRIDEAS (CSN5), and ATLAS (CSN1). Irradiation studies at the TRIGA reactor in Ljubljana (Slovenia) have received funding from the European Commission under the FP7 Research Infrastructures project AIDA, grant agreement no. 262025. 


\section{References}

[1] M. Da Rold, et al., Study of breakdown effects in silicon multiguard structures, IEEE Trans. Nucl. Sci. NS-46(4) (1999) 1215.

[2] The ATLAS Collaboration, Letter of Intent for the Phase-II Upgrade of the ATLAS Experiment, CERN-2012-022, LHCC-I-023, 2012.

[3] The ATLAS IBL Collaboration, Prototype ATLAS IBL Modules using the FE-I4A Front-End Readout Chip, JINST 7 (2012) P11010.

[4] G. Ruggiero, et al., Planar edgeless silicon detectors for the TOTEM experiment, IEEE Trans. Nucl. Sci. NS-52 (5) (2005) 1899.

[5] The ATLAS Collaboration, Letter of Intent for the Phase-I Upgrade of the ATLAS Experiment, CERN-LHCC-2011-012, LHCC-I-020, 2011.

[6] J. Jakubek, et al., Large Area Pixel Detector With Full Area Sensitivity Composed of 100 Timepix Assemblies With Edgeless Sensors, JINST 9 (2014) C04018.

[7] T.E. Hansen, et al., Edge-on Detectors with Active Edge for X-Ray Photon Counting, in Conference Record of 2011 IEEE Nuclear Science Symposium, Paper N25-6.

[8] Z. Li, et al., Electrical and transient current characterization of edgeless Si detectors diced with different methods, IEEE Trans. Nucl. Sci. NS- 49(3) (2002) 1040.

[9] C.J. Kenney, et. al., Results from 3-D silicon sensors with wall electrodes: near-cell-edge sensitivity measurements as a preview of active-edge sensors, IEEE Trans. Nucl. Sci. NS-48(6) (2001) 2405.

[10] C.J. Kenney, et al., Active-edge planar radiation sensors, Nucl. Instrum. Methods A 565 (2006) 272.

[11] C.J. Kenney, et al., Use of active-edge silicon detectors as X-ray microbeam monitors, Nucl. Instrum. Methods A 582 (2007) 178.

[12] S. I. Parker, et al., 3DX: an X-ray pixel array detector with active edges, IEEE Trans. Nucl. Sci. NS53(3) (2006) 1676.

[13] C. Da Via, et al., 3D active edge silicon detector tests with 120 GeV muons, IEEE Trans. Nucl. Sci. NS- 56(2) (2009) 505.

[14] S. H. Christiansen, et al., Wafer direct bonding: from advanced substrate engineering to future applications in micro/nanoelectronics, Proc. of the IEEE 94 (2006) 2060.

[15] C. Da Via, et al., 3D Silicon Sensors: Design, Large Area Production and Quality Assurance for the ATLAS IBL Pixel Detector Upgrade, Nucl. Instrum. Methods A 694 (2012) 321.

[16] M. Povoli, et al., Development of planar detectors with active edge, Nucl. Instrum. Methods A 658 (2011) 103.

[17] J. Kalliopuska, et al., Alternative fabrication process for edgeless detectors on 6 in. wafers, Nucl. Instrum. Methods A 633 (2011) S50.

[18] X. Wu, et al., Recent advances in processing and characterization of edgeless detector, JINST 7 (2012) C02001.

[19] A. Macchiolo, et al., Active edge pixel sensors and development of four-side buttable modules using vertical integration technologies, presented at HSTD9, Hiroshima 2013, to appear in NIM A. 
[20] M. Bomben, et al., Novel silicon n-on-p edgeless planar pixel sensors for the ATLAS upgrade, Nucl. Instrum. Methods A 730 (2013) 215.

[21] M. Bomben, et al., Performance of Irradiated Thin Edgeless N-on-P Planar Pixel Sensors for ATLAS Upgrades, in Conference Record of 2013 IEEE Nuclear Science Symposium, Paper N13-2.

[22] M. J. Bosma, et al., Edgeless silicon sensors for Medipix-based large-area X-ray imaging detectors, JINST 6 (2011) C01035.

[23] V. Fadeyev, et al., Scribe-cleave-passivate (SCP) slim edge technology for silicon sensors, Nucl. Instrum. Methods A 731 (2013) 260.

[24] V. Fadeyev, et al., Scribe-Cleave-Passivate (SCP) Slim Edge Technology, presented at HSTD9, Hiroshima 2013, to appear in NIM A.

[25] S. Kamada, et al., Development of HPK $n$ in $p$ Pixel Sensors for HL-LHC, presented at HSTD9, Hiroshima 2013, to appear in NIM A.

[26] G.-F. Dalla Betta, et al., Development of Active and Slim Edge Terminations for 3D and Planar Detectors, in Conference Record of 2011 IEEE Nuclear Science Symposium, Paper N25-4.

[27] M. Povoli, et al., Functional characterization of planar sensors with active edges using laser and XRay beam scans, Nucl. Instrum. Methods A 718 (2013) 350.

[28] M. Povoli, et al., Slim edges in double-sided silicon 3D detectors, JINST 7 (2012) C01015.

[29] M. Povoli, et al., Design and testing of an innovative slim-edge termination for silicon radiation detectors, JINST 8 (2013) C11022.

[30] M. Povoli, et al., Layout and Process Improvements to Double-Sided Silicon 3D Detectors Fabricated at FBK, in Conference Record of 2012 IEEE Nuclear Science Symposium, Paper N14204. 